\title{
Implementation of Rational Function Model for Rad- Orthokit Generation on Cartosat-1 Data
}

\author{
K. Sudhakar, PhD \\ NRSC, ISRO/DOS
}

\author{
P. Abhiram \\ BITS Pilani
}

\author{
S. Murali Krishnan, PhD \\ NRSC, ISRO/DOS
}

\author{
T. Jayasudha \\ NRSC, ISRO/DOS
}

\author{
P. Shashivardhan Reddy \\ NRSC, ISRO/DOS
}

\author{
G. Pranay Raj \\ BITS Pilani
}

\author{
M. Manju Sarma \\ NRSC, ISRO/DOS
}

\author{
B. Lakshmi \\ NRSC, ISRO/DOS
}

\begin{abstract}
Sensor models are required to establish the relationship between 3D object space and 2D image space. Traditionally this is done using the physical sensor model where the complete parameters of physical imaging system are known. The replacement sensor models are required to establish this relation without the knowledge of the physical sensor model. The rational function model (RFM) is one of the replacement model used in remote sensing with 78 rational polynomial coefficients (RPCs). RFM is a complete mathematical model, which approximately describes the physical imaging process in photogrammetry and remote sensing. In the absence of interior and exterior orientation such as camera model, position and orientation information of specific sensor, large number of ground control points (GCPs) are needed to solve all the unknown coefficients of the RFM and to achieve higher accuracies in the photogrammetric processing. The rational function model(RFM) can be used either as a replacement for physical sensor model ( terrain dependent) or to express the physical model in the form of RPCs ( terrain independent) for further processing.
\end{abstract}

In this paper the implementation aspects of terrain dependent RFM model for Cartosat-1 data for the Chitrapur, Simla, Himachal Pradesh state, India and the accuracies achieved and the stability of the model are discussed. The direct least square solutions to the RFM are implemented using row reduction. The validation of RFM is done at check points and achieved planimetric accuracy $1.5 \mathrm{~m}, 3.38 \mathrm{~m}$ with respect to CE90 in $\mathrm{X}$ and $\mathrm{Y}$ directions respectively.

\section{General Terms}

Check Points ,Ground Control Points, RMSE.

\section{Keywords}

Feature editing, RFM, row reduction, Sensor Model,CE90.

\section{INTRODUCTION}

A sensor model describes the geometric relationship of 3D object coordinates to $2 \mathrm{D}$ image coordinates. There are various models to provide the relation and each model has its own merits and demerits. There are two broad categories of sensor models, which are generalized and physical. A physical sensor model needs the physical parameters of the sensor such as the position and the orientation of the sensor with respect to an object space co-ordinate system and these parameters are not correlated and yield accurate results. But in the recent past the satellite data providers are not ready to part with the sensor model. As an alternative remote sensing data providers started RPC models where sensor model is provided in the form of RPC coefficients. But when RPCs are not provided by the data suppliers generalized terrain dependent methods are to be used to carry out ortho-rectification of the data. The generalized models represent the transformation of the coordinates from $3 \mathrm{D}$ to $2 \mathrm{D}$ by rational functions or polynomials. This paper describes implementation of RFM to establish relationship between object space and image space for Cartosat-1 data.

\section{THE RATIONAL FUNCTION MODEL}

RFM is a mathematical transformation between object space and image space co-ordinates. The RFM is defined as ratio of two cubic polynomials separately for row and column coordinates of image and hence it is given in[3] as

$$
\begin{aligned}
& r=\frac{N_{r}(X, Y, Z)}{D_{r}(X, Y, Z)} \\
& c=\frac{N_{c}(X, Y, Z)}{D_{c}(X, Y, Z)} 1
\end{aligned}
$$

Where $\mathrm{r}, \mathrm{c}$ are normalized row and column coordinates of image space and $\mathrm{X}, \mathrm{Y}, \mathrm{Z}$ are normalized coordinates of object space. The constant term in the denominator of $r$ and $c$ is taken as 1 to avoid singularity in equation(1). Also $\operatorname{Nr}(X, Y, Z), \operatorname{Dr}(X, Y, Z), N c(X, Y, Z), D c(X, Y, Z)$ defined as in[3]

$$
\begin{aligned}
\operatorname{Nr}(X, Y, Z)=a_{1}+ & a_{2} X+a_{3} Y+a_{4} Z+a_{5} X Y+a_{6} X Z+a_{7} Y Z \\
& +a_{8} X^{2}+a_{9} Y^{2}+a_{10} Z^{2}+a_{11} X Y Z \\
& +a_{12} X^{3}+a_{13} X Y^{2}+a_{14} X Z^{2}+a_{15} X^{2} Y \\
& +a_{16} Y^{3}+a_{17} Y Z^{2}+a_{18} Z X^{2}+a_{19} Z Y^{2} \\
& +a_{20} Z^{3} \\
\operatorname{Dr}(X, Y, Z)=b_{1}+ & b_{2} X+b_{3} Y+b_{4} Z+b X Y+b X Z+b Y Z \\
& +b_{8} X^{2}+b_{9} Y^{2}+b_{10} Z^{2}+b_{11} X Y Z \\
& +b_{12} X^{3}+b_{13} X Y^{2}+b_{14} X Z^{2}+b_{15} X^{2} Y \\
& +b_{16} Y^{3}+b_{17} Y^{2}+b_{18} Z X^{2}+b_{19} Z Y^{2} \\
& +b_{20} Z^{3} \\
& \\
& b_{1}=1
\end{aligned}
$$




$$
\begin{aligned}
\mathrm{Nc}(X, Y, Z)=c_{1}+ & c_{2} X+c_{3} Y+c_{4} Z+c_{5} X Y+c_{6} X Z+c_{7} Y Z \\
& +c_{8} X^{2}+c_{9} Y^{2}+c_{10} Z^{2}+c_{11} X Y Z \\
& +c_{12} X^{3}+c_{13} X^{2}+c_{14} X^{2}+c_{15} X^{2} Y \\
& +c_{16} Y^{3}+c_{17} Y^{2}+c_{18} Z^{2}+c_{19} Z Y^{2} \\
& +c_{20} Z^{3}
\end{aligned}
$$

$$
\begin{aligned}
\operatorname{Dc}(X, Y, Z)=d_{1}+ & d_{2} X+d_{3} Y+d_{4} Z+d_{5} X Y+d_{6} X Z+d_{7} Y Z \\
& +d_{8} X^{2}+d_{9} Y^{2}+d_{10} Z^{2}+d_{11} X Y Z \\
& +d_{12} X^{3}+d_{13} X Y^{2}+d_{14} X Z^{2}+d_{15} X^{2} Y \\
& +d_{16} Y^{3}+d_{17} Y^{2}+d_{18} Z X^{2}+d_{19} Z Y^{2} \\
& +d_{20} Z^{3}
\end{aligned}
$$

$\mathrm{d}_{1}=1$

Where $a_{i}^{\prime} s, b_{i}^{\prime} s$, ci $s, d_{i}^{\prime} s$ are polynomial coefficients.

In RFM model, the distortions caused by optical projection can be expressed as $1^{\text {st }}$-order polynomial coefficients, and the error caused by the earth curvature, atmospheric refraction and lens distortion can be corrected by $2^{\text {nd }}$-order polynomial coefficients, and that caused by other unknown distortions can be simulated by $3^{\text {rd }}$-order polynomial coefficients[2].

\subsection{RFC Generation Methodology}

In order to avoid time consuming process and to improve the numerical stability of the equations in 1, both image and object space co-ordinates are normalized to the range of -1.0 to 1.0 [1]. The normalization of the coordinates is computed as follows[1]:

$$
r_{n}=\frac{r-r_{o}}{r_{s}}, \quad c_{n}=\frac{c-c_{o}}{c_{s}}
$$

$\mathrm{X}_{\mathrm{n}}=\frac{\mathrm{X}-\mathrm{X}_{0}}{\mathrm{X}}, \mathrm{Y}_{\mathrm{n}}=\frac{\mathrm{Y}-\mathrm{Y}_{\mathrm{o}}}{\mathrm{Y}_{\mathrm{s}}}, \mathrm{Z}_{\mathrm{n}}=\frac{\mathrm{Z}-\mathrm{Z}_{0}}{\mathrm{Z}_{\mathrm{s}}}$.

Where, $r_{0}, c_{0}, X_{0}, Y_{0}, Z_{0}$ are themean values for scan line number, pixel number, latitude, longitude and height respectively, and $r_{s}, c_{s}, X_{s}, Y_{s}, Z_{s}$ are the scale values for scan line number, pixel number, latitude, longitude and height respectively. So from the expression of scan line and pixel line functions, we have [1]

$$
\begin{aligned}
& r=\frac{\left(1 X Y Z \ldots Y^{3} Z^{3}\right) \cdot\left(a_{1} a_{2} \ldots a_{20}\right)^{T}}{\left(1 X Y Z \ldots Y^{3} Z^{3}\right) \cdot\left(1 b_{2} \ldots b_{20}\right)^{T}} \\
& c=\frac{\left(1 X Y Z \ldots Y^{3} Z^{3}\right) \cdot\left(c_{1} c_{2} \ldots c_{20}\right)^{T}}{\left(1 X Y Z \ldots Y^{3} Z^{3}\right) \cdot\left(1 d_{2} \ldots d_{20}\right)^{T}}
\end{aligned}
$$

Where $\mathrm{a}_{\mathrm{i}}{ }^{\prime} \mathrm{sb}_{\mathrm{i}}{ }^{\prime} \mathrm{sc}_{\mathrm{i}}{ }^{\prime} \mathrm{sd}_{\mathrm{i}}$ 'sare polynomial coefficients.

$$
\text { Let } M=\left[\begin{array}{cccc}
1 & X_{1} & & -r_{1} Z_{1}^{3} \\
1 & X_{2} & \cdots & -r_{n} Z_{2}^{3} \\
& \vdots & \ddots & \vdots \\
1 & X_{3} & \cdots & -r_{n} Z_{n}^{3}
\end{array}\right]
$$

$\mathrm{R}=(\mathrm{X} 1, \mathrm{X} 2, \ldots, \mathrm{Xn})$ is set of $\mathrm{n}$ number of ground control points. $J=\left(a_{0} a_{1} \cdots a_{19} b_{1} b_{2} \cdots b_{19}\right)^{T}$ is the result matrix containing the RFCs for row of image coordinates.

We get, from equation number(1)as in [1]

$$
\mathrm{V}=\mathrm{MJ}-\mathrm{R} \rightarrow(2)
$$

Where $\mathrm{V}$ is the error matrix. Since the error to be minimum, first take the values of $\mathrm{V}$ to be zero for direct least square solution[1]. It is noticed that solving equation(2) normally is critical because the matrix size is large and hence to solve the above equation multiply both the sides of the equation by the inverse of $\mathrm{M}$ matrix in order to get square matrices which can be worked easily with in terms of taking inverse and hence the equation becomes

$$
0=\left(M^{T} M\right) J-M^{T} R
$$

Simplifying it further gives

$$
J=\left(M^{T} M\right)^{-1} M^{T} R
$$

Inverse of $\mathrm{M}^{\mathrm{T}} \mathrm{M}$ is found with row reduction method by $\mathrm{C}$ code and then computed the rational polynomial coefficients for a particular scene of Cartosat -1 from a set of ground control points.

\subsection{Dataset used and Implementation}

Cartosat-1 data acquired during the period 10th March,2009 over Chitrapur, Simla, Himachal Pradesh state, India where the terrain undulations vary between 330 to $850 \mathrm{~m}$ and ground control points which have an accuracy of $1 \mathrm{~m}$ are used for generation of RPC's. The 600 GCPs are identified using Leica photogrammetric software. After adjustment with the control points and scales, offsets in image space and object space derived for 600 points and only 387 points are used in RFM model to generate RPC's. A text file is generated for 387 points containing the line, column pixel, latitude, longitude and height respectively. This file is used as an interface to the developed s/w to the RFM model.

The Input data is normalized using line offsets and scales. The variable matrix inverse is described by row reduction method using elementary operation by augmenting the identity matrix with the same dimension of the variable matrix. The same is done for column. 39 RPCS for row and 39 RPCS for column were generated. 


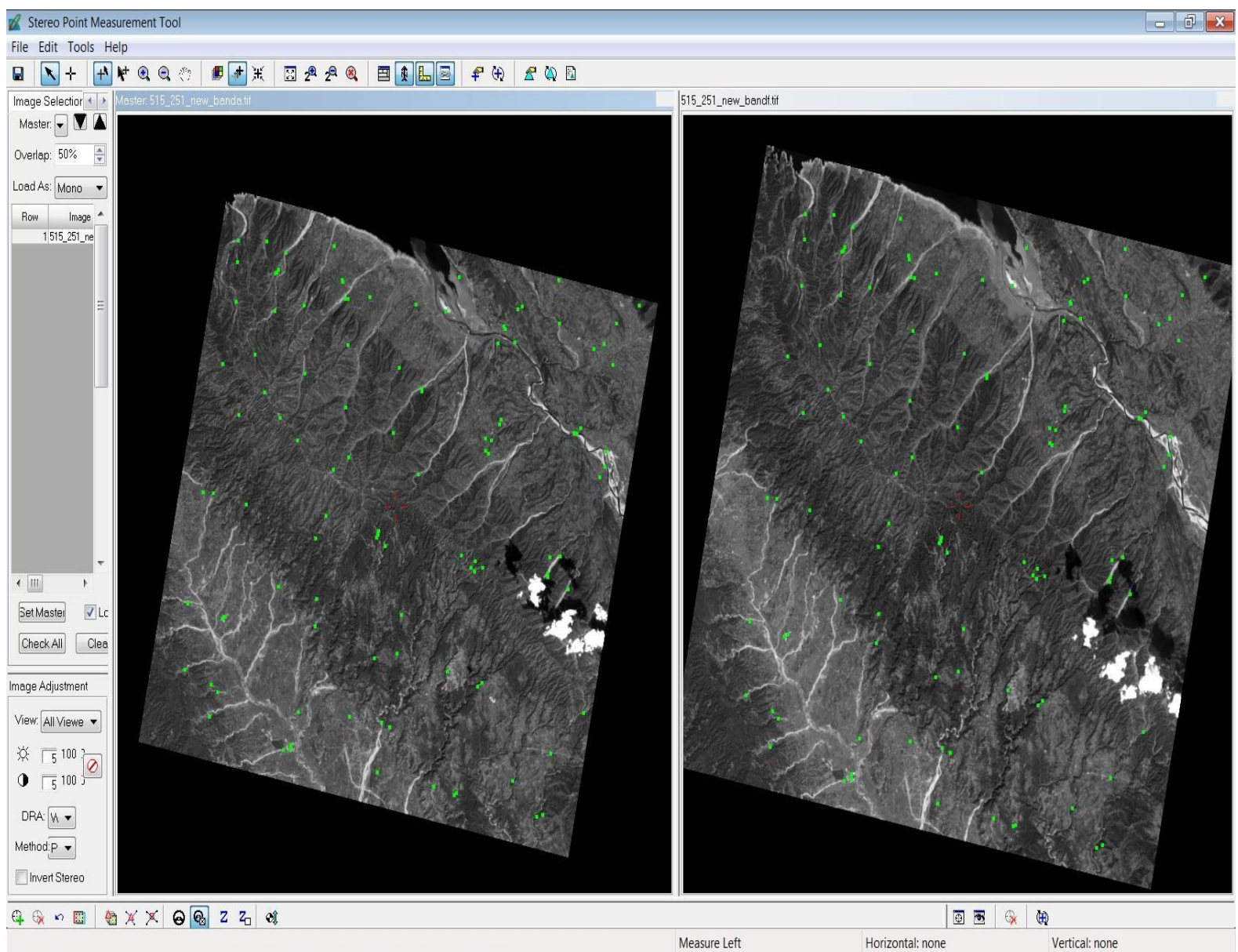

Figure 1:Marking of tie points on stereo pair

\section{VALIDATION OF RFM}

To validate Rational Function Model, the rational polynomial coefficients and the values of latitude, longitude and height of check points are substituted in the equations (1) to get the values of line and pixels back. The errors between the check points image coordinates (line, pixel) and the computed image coordinates of same points using obtained RPC's through RFM model are shown Table1. For total image obtained RMSE $=0.1137580$ pixels and calculated CE90(circular error/planimetric accuracy) at control points, check points with CE90 $=1.5175 *$ RMSE and vertical accuracyLE90 $=1.6449 *$ RMSE are shown in Table2.

Table 1. Differences between actual and calculated image coordinates of check points

\begin{tabular}{|c|c|c|c|c|c|c|}
\hline Check Points Line No's & Check Points Pirel No's & & $\begin{array}{l}\text { Obtained } \\
\text { Line No's }\end{array}$ & $\begin{array}{l}\text { Obtained } \\
\text { Pixel No's }\end{array}$ & $\begin{array}{c}\text { Difference } \\
\text { of Lines }\end{array}$ & $\begin{array}{c}\text { Difference } \\
\text { of Pixels }\end{array}$ \\
\hline 387.594196 & 477.448157 & 1 & 387.6063 & 477.5092 & -0.01209 & -0.06102 \\
\hline 755.371459 & 405.801379 & 2 & 755.4197 & 405.8771 & -0.04819 & -0.07572 \\
\hline 1122.864876 & 332.88501 & 3 & 1122.849 & 332.9274 & 0.016291 & -0.04238 \\
\hline 1490.90964 & 262.335203 & 4 & 1490.871 & 262.3728 & 0.039004 & -0.0376 \\
\hline 1858.735615 & 190.837214 & 5 & 1858.732 & 190.8752 & 0.003484 & -0.03797 \\
\hline 2226.667735 & 119.927684 & 6 & 2226.658 & 119.9675 & 0.009549 & -0.03982 \\
\hline 115.179527 & 932.798355 & 7 & 115.1485 & 932.8159 & 0.03107 & -0.01753 \\
\hline 483.036669 & 861.241314 & 8 & 483.0476 & 861.2563 & -0.01092 & -0.01504 \\
\hline 850.690214 & 788.79148 & 9 & 850.5924 & 788.8009 & 0.097846 & -0.00943 \\
\hline 1218.607123 & 717.383069 & 10 & 1218.55 & 717.3751 & 0.057041 & 0.008004 \\
\hline 1586.415846 & 645.433877 & 11 & 1586.418 & 645.4467 & -0.00217 & -0.01281 \\
\hline 1954.325819 & 573.962853 & 12 & 1954.332 & 573.9712 & -0.00653 & -0.00833 \\
\hline 2322.467739 & 503.844709 & 13 & 2322.461 & 503.8578 & 0.006574 & -0.01312 \\
\hline 2690.445071 & 433.007933 & 14 & 2690.437 & 433.0215 & 0.007707 & -0.01358 \\
\hline 3058.374649 & 361.986327 & 15 & 3058.362 & 362.0003 & 0.012528 & -0.01395 \\
\hline 3426.301548 & 291.164262 & 16 & 3426.284 & 291.1792 & 0.017566 & -0.01493 \\
\hline 3794.161441 & 219.932388 & 17 & 3794.178 & 219.9391 & -0.0162 & -0.00668 \\
\hline 4162.006206 & 148.726358 & 18 & 4162.012 & 148.7333 & -0.00625 & -0.00692 \\
\hline 4529.794365 & 77.013266 & 19 & 4529.798 & 77.01804 & -0.00334 & -0.00477 \\
\hline 210.422471 & 1316.029239 & 20 & 210.3445 & 1316.051 & 0.078017 & -0.0219 \\
\hline 578.463045 & 1244.88674 & 21 & 581.6181 & 1244.908 & -3.15502 & -0.02101 \\
\hline 946.052818 & 1171.847475 & 22 & 946.0258 & 1171.861 & 0.026981 & -0.01365 \\
\hline
\end{tabular}




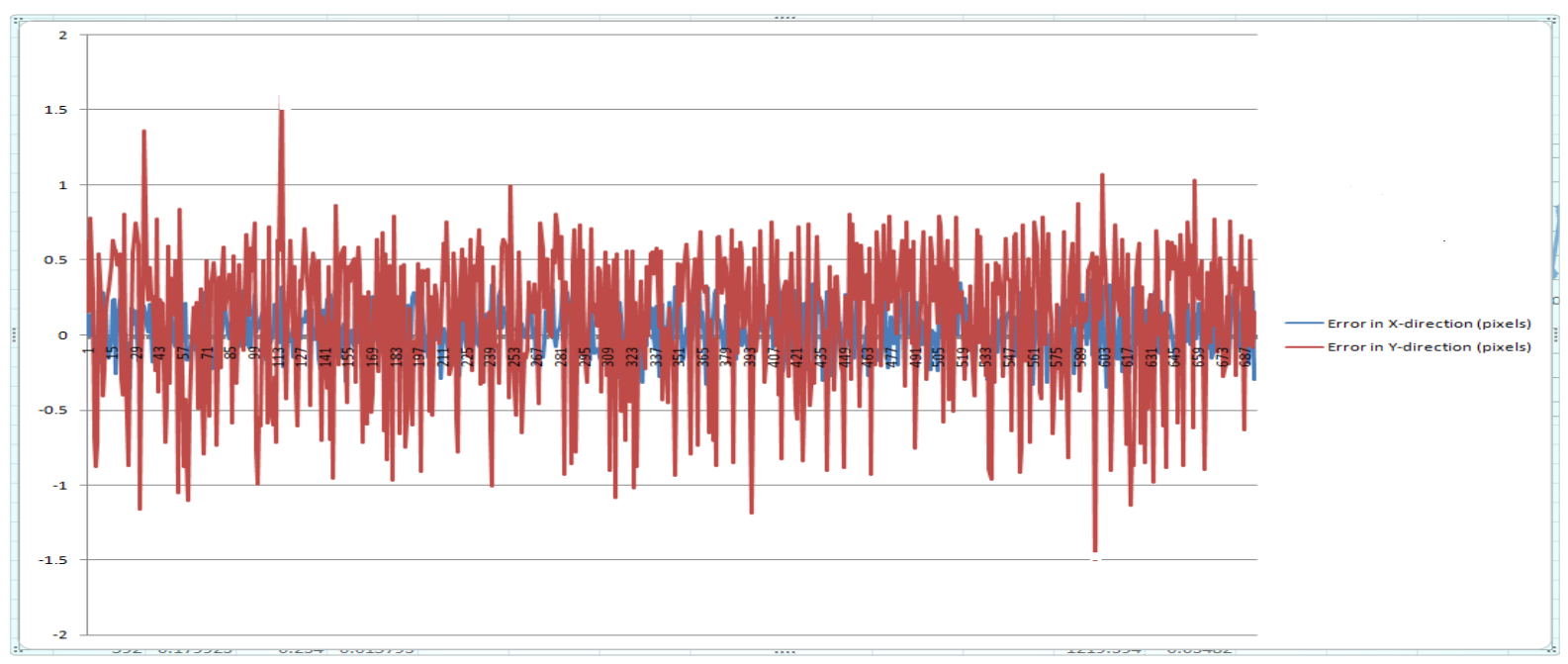

Figure 2: RPC accuracy in pixels.

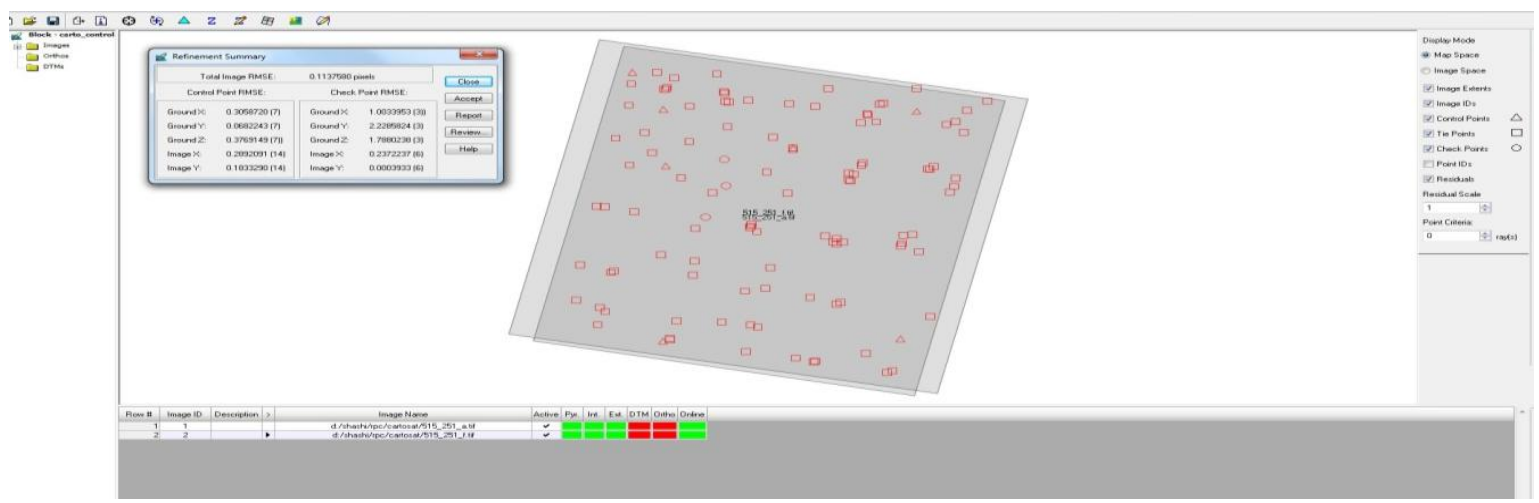

Figure 3: RMSE of total image, control points, check points.

Table 2. CE90 and LE90 values.

\begin{tabular}{|c|c|c|c|c|c|c|}
\hline & & & \multicolumn{2}{|c|}{ Control Points } & \multicolumn{2}{c|}{ Check points } \\
\cline { 5 - 7 } & $\begin{array}{c}\text { Control Points } \\
\text { RMSE }\end{array}$ & $\begin{array}{c}\text { Check Points } \\
\text { RMSE }\end{array}$ & CE90 & LE90 & CE90 & LE90 \\
\cline { 5 - 7 } & & & & & & \\
\hline Ground X & $0.30587206(7)$ & $1.0033953(3)$ & 0.464160851 & - & 1.522652368 & - \\
\hline Ground Y & $0.0682243(7)$ & $2.2285824(3)$ & 0.103530375 & - & 3.381873792 & - \\
\hline Ground Z & $0.3769149(7)$ & $1.7880238(3)$ & - & 0.619987 & - & 2.941120 \\
\hline
\end{tabular}

\subsection{Conclusions}

Approaches for Cartosat-1 Orthokit product generation are presented including the test results for the Datasets used. From the tests performed on the given datasets, it can be inferred that RPCs can be provided to the users for photogrammetric processing at their end without compromising on the accuracy when sufficient GCP'S are available. The Rational Function model is able to model all the distortions in the image and achieved planimetric accuracy $1.5 \mathrm{~m}, 3.38 \mathrm{~m}$ in $\mathrm{X}$ and $\mathrm{Y}$ directions with respect to CE90 respectively and vertical accuracy is up to $3 \mathrm{~m}$ with respect to LE90 at check points.

\subsection{Acknowledgments}

We would like to thanks to the DD/DPPA\&WAA, NRSC for his ideal guidance and Director, NRSC for cordial support .

\section{REFERENCES}

[1] C.Vincent Tao and Young Hu "A Comprehensive study on the rational function model for photogrammetric processing”, PE \&RS, 67(12) 2001, pp 1347-1357.

[2] S.J.Liu,X.H Tong "Transformation between rational functional model and rigorous sensor model for high resolution satellite imagery" the international archives of photogrammetry, remote sensing and spatial and information sciences vol 37,partB1, Beijing(2008).

[3] Tengfei Long,Weili JLAO “An automatic selection and solving method for rational polynomial coefficients based on nested regression" the 33rda Asian conference on remote sensing. 